\title{
Capacidades organizacionales generadoras de valor: análisis del sector industrial
}

\section{Organizational capacities to generate value: analysis of industrial sector}

\author{
Glenda Ponce-Espinosa \\ Universidad Técnica Particular de Loja. Ecuador \\ geponce@utpl.edu.ec \\ orcid.org/0000-0002-5781-9848 \\ Diana L. Espinoza \\ Universidad Técnica Particular de Loja. Ecuador \\ dlespinoza@utpl.edu.ec \\ orcid.org/0000-0002-8035-1278 \\ Julio Ríos-Zaruma \\ Universidad Técnica Particular de Loja. Ecuador \\ jarios@utpl.edu.ec \\ orcid.org/0000-0002-8271-9398 \\ Karla Gabriela Tapia C. \\ Universidad Técnica Particular de Loja. Ecuador \\ kgtapia@utpl.edu.ec \\ orcid.org/0000-0002-9116-2181
}

\begin{abstract}
Resumen
El propósito de esta investigación es determinar las capacidades organizacionales que han sido desarrolladas por las empresas del sector industrial de la ciudad de Loja, a partir del modelo de las 7s de Mckinsey propuesto por Tom Peters y Robert Waterman. Se aplicó el cuestionario como instrumento para la recolección de datos a la población objeto del estudio que son las empresas que pertenecen a la Cámara de Industrias de Loja, los resultados obtenidos fueron comparados con la variación de la rentabilidad y el impuesto a la renta causado en el periodo 2013-2014. El estudio permitió determinar que las empresas presentaron variabilidad positiva durante el periodo mencionado, aplicaron un estilo de liderazgo autocrático, flujo de información descendente, estructura organizacional definida así como misión y visión. El tipo de innovación más común fue en productos y no aplicaron instrumentos que permitan determinar el clima laboral. Por otro lado, las empresas que no presentaron variabilidad positiva se caracterizaron por un estilo de liderazgo liberal y democrático, flujo de información multidireccional e innovación reactiva.
\end{abstract}

\begin{abstract}
The purpose of this research is determinate the organizational capacities that have been developed by the companies of the industrial sector of the city of Loja, based on the Mckinsey 7s model proposed by Tom Peters and Robert Waterman. The questionnaire was applied as an instrument for the collection of data to the population of the study that are the companies that belong to the Cámara de Industrias de Loja; the results obtained were compared with the variability in profitability and the income tax caused in the period 2013-2014. The study allowed to determine that companies presented positive variability during the mentioned period applied a style of autocratic leadership, downward flow of information, defined organizational structure as well as mission and vision, the most common type of innovation was in products and did not apply instruments that allow Determine the working environment. On the other hand, companies that did not present positive variability were characterized by a style of liberal and democratic leadership, multidirectional information flow, reactive innovation and to determine the work climate used surveys.
\end{abstract}

\section{Palabras clave | keywords}

Capacidades organizacionales, 7s Mckinsey, estilo de liderazgo, estrategia, estructura, flujo de información. Organizational capacities, 7s Mckinsey, leadership style, strategy, structure. information flow 


\section{Introducción}

Las organizaciones se ven expuestas a un intercambio de energía e información con el entorno, puesto que se encuentran en constante interacción con el mismo. Sin embargo, cuanto mayor sea el cambio en el entorno de una empresa, más debe procurar basar su estrategia en los recursos y capacidades internas en lugar de hacerlo en el mercado. Es por esta razón que uno de los grandes retos que enfrentan los gerentes corresponde a la habilidad para alinear a la estrategia empresarial los esfuerzos que se realizan en las organizaciones con la finalidad de lograr mayor efectividad en las operaciones de las mismas.

O'Reilly y Thusman (2013) han realizado una recopilación de definiciones para esta habilidad, determinándola como "la capacidad de la empresa para integrar, construir y reconfigurar las competencias internas y externas para abordar los cambios rápidos del ambiente" o como "la capacidad de una organización para crear, ampliar, modificar su base de recursos" (O’Reilly \& Tushman, 2013).

Los recursos y las capacidades se han convertido en la base para la estrategia a largo plazo de la empresa. Estos elementos proveen la dirección básica y son la fuente principal de la rentabilidad para la empresa. A partir de este principio, el modelo de las 7s de McKinsey sostiene que los cambios en la eficacia de una empresa son directa consecuencia de la interacción de múltiples factores, muchos de los cuales no son obvios o no han sido considerados en los modelos tradicionales. Esta investigación considera que además de los factores internos es importante conocer cuáles son las capacidades de la organización que inciden en la creación de valor del negocio así como cuál es la combinación de los elementos del modelo de McKinsey que permite a las organizaciones generar valor.

Este modelo muestra que la organización va más allá de sus objetivos. Se refiere a una organización cuya eficiencia la centra en la interacción de estos elementos, considerando que la empresa eficaz no está representada precisamente por un modelo único, cerrado y estable en el tiempo, sino más bien constituye un sistema abierto, permeable y condicionado a la influencia permanente de una serie de factores de contingencia y a la visión de sus directivos o administradores acerca de la forma cómo debe ser dirigida y organizada. Por esta razón, adquiere especial importancia el enfoque estratégico que responde a la necesidad de formular una visión corporativa que permita a la organización 
dar una adecuada respuesta (la estrategia) relativa a las decisiones más convenientes sobre las actividades a desarrollar o sobre los negocios que desempeña o puede desempeñar (Suárez-Hernández \& Mirón, 2013).

En este sentido, el presente artículo está organizado en dos secciones: la primera sección desarrolla el concepto de capacidad organizacional y explica detalladamente el modelo de las 7s, así como la descripción de los elementos que la conforman, especificando la administración efectiva de los factores que permiten comprender la naturaleza y características de la parte conceptual. Una segunda sección establece el modelo de Mckinsey dentro de las empresas sujetas al estudio. Este proceso está soportado por los siete elementos delimitados: estrategia, estructura, sistema, estilo, personal, habilidades y valores compartidos.

El modelo de las 7s de Mckinsey se plantea bajo la premisa de que "una organización no es solamente estructura", sino que basa su propuesta en siete elementos -incluyendo lógicamente estructura-, a saber: "structure, systems, style, staff, skills, strategy, shared values"; aspectos internos que la organización necesita alinear a fin de lograr éxito (Peters $\&$ Waterman, 1980). Los creadores de este modelo diferencian y proponen una clasificación de los elementos duros (estrategia, estructura y sistema) y suaves (estilo, personal, habilidades y valores compartidos).

Con el fin de comprender el planteamiento teórico, los siete elementos se componen por:

- Estrategia: Se refiere a los planes anticipados o en respuesta a los cambios del entorno. Se refiere a la ruta que ha elegido la organización para seguir. La estrategia de la organización se ve reflejada en la misión y la visión que se refiere al camino al cual se dirige la empresa a largo plazo. La estrategia, la misión, visión y valores de las organizaciones son consideradas parte de la identidad de la organización y son además el elemento distintivo y la expresión de la personalidad de la organización (Martínez, Pérez, \& Rodríguez del Bosque, 2014).

- Una vez que se han definido metas y estrategias que permitan lograr los objetivos de la organización, el gerente debe asegurar que la estructura se ajusta a la estrategia (Ivanko, 2013), es así que el diseño y la asignación de tareas a los miembros de la organización corresponde al siguiente elemento de este modelo.

- Estructura: Trata sobre la especialización y coordinación de actividades, influenciada principalmente por la estrategia y el tamaño de la organización y su diversidad. 
- La estructura organizacional es definida como el grado en que las actividades de una organización son estandarizados y en el que las normas y procedimientos guían el comportamiento de los empleados (Robbins \& Coulter, 2005). Las organizaciones, además de la estructura organizacional declarada, requieren de un nivel de formalización que implique el establecimiento de reglas, procedimientos, y documentación escrita como manuales de políticas y descripción de puestos que establecen los deberes y derechos de los empleados (Daft, 2012).

- Sistema: Compuesto por los procedimientos formales e informales que se lleva a cabo en las organizaciones. Los sistemas para su funcionamiento requieren de estos aspectos: adaptación (debe abarcar las situaciones externas, adaptarse a su entorno y adaptar el entorno a sus necesidades), capacidad de alcanzar las metas (debe definir y alcanzar sus metas fundamentales), integración (regular la interrelación entre sus componentes), mantenimiento de patrones (proporcionar, mantener y renovar la motivación de los individuos y las pautas culturales que lo integran) (Parsons, 1937).

- La cultura organizacional: Que forma parte también de los sistemas y se refieren a los valores, creencias y normas dominantes desarrolladas a lo largo de la vida de la organización. Estos elementos influyen en el comportamiento de los empleados (Reis \& Azebedo, 2015).

- Estilo de administración: Existe una relación cercana entre la forma de pensar y la forma de actuar en las organizaciones. Muchos de los problemas que surgen en la organización tienen su raíz en esta forma de pensamiento, sin embargo, éstas pueden dar lugar a nuevas y mejoradas formas de organización (Ivanko, 2013).

- El liderazgo: Definido como "El arte o proceso de influir en las personas para que se esfuercen voluntaria y entusiastamente en el cumplimiento de metas grupales" (Koontz \& Weihrich, 2004); se refiere al estilo de administración, el cual se ve influido por el estilo de liderazgo que se aplica dentro de la organización.

- Así, el liderazgo presenta diferentes estilos que pueden ser adoptados y aplicados en las organizaciones. Estos estilos se los considera como las teorías que estudian el liderazgo en términos de formas de comportamiento del líder en relación con sus subordinados; es decir las maneras en que el líder orienta su conducta (Koontz \& Weihrich, 2004) clasifican los estilos de liderazgo en: autocrático (quien impone y espera cumplimiento, es dogmático y seguro y conduce por medio de la capa- 
cidad denegar u otorgar premios o castigos) democrático (consulta con sus subordinados respecto de acciones y decisiones probables, y alienta su participación) y liberal (hace un uso muy reducido de su poder, en caso de usarlo, ya que les concede a sus subordinados un alto grado de independencia en sus acciones).

- Personal: Que se refiere al capital humano de la organización y a cómo éste es desarrollado, entregado, socializado, integrado y motivado y a la manera en que sus carreras son administradas. (Ivanko, 2013). Respecto a este aspecto, su importancia radica en la manera en que el personal de la organización es comunicado, entendiéndose por comunicación organizacional a la comunicación formalmente definida en la organización para procesos específicos internos y en relación con el entorno. El personal requiere ser informado de los aspectos que suceden en la organización, con los clientes, los proveedores, distribuidores, socios estratégicos.

- Habilidades: Representan las competencias distintivas, es decir, lo que la organización realiza mejor y que contribuye al logro de la misión y la visión propuestas por la organización.

- Valores compartidos: Compuestos por los elementos que influyen en el grupo de trabajo a fin de lograr alcanzar los objetivos comunes. Se los considera como los conceptos y principios rectores de la organización, son aspectos que van más allá de las declaraciones de los objetivos organizacionales

\section{Material y métodos}

El presente estudio es de tipo descriptivo con un enfoque cualitativo. La población objeto del estudio son las empresas que pertenecen a la Cámara de Industrias de Loja (Ecuador), siendo el cuestionario el instrumento que se utilizó para la recolección de datos, el cual se aplicó a 18 empresas que componen la población, durante el periodo comprendido entre el año 2014 y 2015.

Entre las variables a utilizar, se considera como variable independiente a la variación en la utilidad obtenida a partir del impuesto a la renta causado de la empresa (valor generado) y como variable dependiente a los siete elementos del modelo $7 \mathrm{~S}$ propuesto por McKinsey (capacidades de las organizaciones). 
a. Recolección de datos

Se utilizó un cuestionario que incluye 14 indicadores que son los que permiten la identificación de los elementos del modelo como herramienta para recolección de datos, el cual fue aplicado a los gerentes de las empresas de la Cámara de Industrias de Loja. Este cuestionario está basado en el modelo 7S propuesto por McKinsey, el cual establece los siete elementos explicados ut supra: estrategia, estructura, sistema, estilo de administración, personal, habilidades, valores compartidos y utiliza catorce indicadores que de acuerdo a la literatura revisada, permite identificar los factores cualitativos respecto del modelo.

Para determinar la variación de la utilidad de la empresa, se utilizó como fuente de información a las base de datos del Servicio de Rentas Internas y la Superintendencia de Compañías y se utilizaron los datos de la rentabilidad y el impuesto a la renta causado que se registra en el periodo 2013-2014 por cada una de las empresas como mecanismo para determinar su variación.

Se utilizó esta variable debido a que de forma práctica demuestra la variación de utilidades como indicador financiero de rendimiento. En el periodo de estudio se han seleccionado aquellas que han reportado aumento o disminución en su impuesto a la renta, lo cual indica una tendencia hacia un mejor o menor rendimiento. Aquellas que no muestran variación no se consideraron en el análisis de la Tabla 4: características de los elementos del modelo de Mckinsey de las empresas de acuerdo a su rendimiento.

b. Análisis de datos

Se identificó el factor cualitativo existente para cada empresa, respecto de las 14 variables planteadas en el cuestionario y se agrupa de acuerdo a cada uno de los siete elementos.

Se identifican las capacidades organizacionales que permiten determinar una variación positiva o negativa respecto de la utilidad generada por la empresa en un año. Se calculó la variación existente entre los dos periodos inmediatamente anteriores en base al impuesto declarado.

De esta manera, se plantea la existencia de dos tipos de empresas:

- Empresas que poseen capacidades organizacionales que representan una variación positiva en la utilidad generada en el año de estudio. Para éstas el procedimiento a utilizar es la selección de 4 empresas que han generado un mayor incremento en su variación de utilidad económica en relación al año anterior. 
- Empresas que poseen capacidades organizacionales que representan una variación negativa en la utilidad generada en el año de estudio, en cuyo caso el procedimiento a utilizar es la selección de 4 empresas que han generado un decrecimiento en su variación de utilidad económica en relación al año anterior.

En base a esta categorización se determina el perfil de los dos tipos empresas planteados de acuerdo a la información registrada en las catorce variables y los 7 elementos del modelo de Mckinsey. Se han identificado también las características en base a la prevalencia de este factor dentro de las empresas del segmento analizado.

\section{Análisis y resultados}

La observación de las capacidades internas de las organizaciones industriales de la ciudad de Loja, a través del modelo de las 7S de McKinsey, permitió identificar las características distintivas de las empresas que generaron utilidad durante los periodos de estudio.

De acuerdo a la clasificación definida por la Comunidad Andina de Naciones, Decisión 702, Artículo 3; se define el tamaño de las empresas de acuerdo al valor bruto de sus ventas anuales. De acuerdo a este antecedente las empresas se clasifican como muestra la Tabla 1, las actividades que desarrollan según lo indicado por el Clasificación Internacional Industrial Unificado -CIIU- de las empresas sujetas de estudio, se muestran en la Tabla 2.

Tabla 1. Clasificación por tipo de empresa

\begin{tabular}{|l|l|l|}
\hline \multicolumn{1}{|c|}{ Tipo de empresa } & Número de empresas & Porcentaje \\
\hline $\begin{array}{l}\text { Microempresa } \\
\text { Ventas menores a } 100000\end{array}$ & 2 & $11 \%$ \\
\hline $\begin{array}{l}\text { Pequeña } \\
\text { Ventas entre } 100000 \text { y } 1000.000\end{array}$ & 7 & $39 \%$ \\
\hline $\begin{array}{l}\text { Mediana } \\
\text { Ventas entre 1 000 000 y } 5000000\end{array}$ & 7 & $39 \%$ \\
\hline $\begin{array}{l}\text { Grande } \\
\text { Ventas mayores a } 5000000\end{array}$ & 2 & $11 \%$ \\
\hline & 18 & \\
\hline
\end{tabular}

Fuente: Comunidad Andina de Naciones, Decisión 702, artículo 3 
Tabla 2. Clasificación actividad y sector al que pertenecen

\begin{tabular}{|l|l|l|l|}
\hline \multicolumn{1}{|c|}{ Actividad } & \multicolumn{1}{|c|}{ CIIU } & $\begin{array}{c}\text { Número de } \\
\text { industrias }\end{array}$ & $\begin{array}{c}\text { Sector al que } \\
\text { pertenecen }\end{array}$ \\
\hline Alimentos & C101/C107/C105 & 7 & $39 \%$ \\
\hline Cerámica & C239 & 3 & $17 \%$ \\
\hline $\begin{array}{l}\text { Comercializadora de productos } \\
\text { agrícolas }\end{array}$ & G471 & 1 & $6 \%$ \\
\hline Generación de electricidad & D351 & 1 & $6 \%$ \\
\hline Industria de vidrio & C231 & 1 & $6 \%$ \\
\hline Licores y bebidas & C110 & 2 & $11 \%$ \\
\hline Elaboración de combustibles & C192 & 1 & $6 \%$ \\
\hline Editoriales & C181 & 2 & $11 \%$ \\
\hline TOTAL & & 18 & $100 \%$ \\
\hline
\end{tabular}

Fuente: Investigación propia

Las características que describen estos elementos del modelo de McKinsey se señalan en la Tabla 3.

\section{Tabla 3. Características de los elementos del Modelo de Mckinsey}

\begin{tabular}{|c|c|c|}
\hline $\begin{array}{c}\text { Tipo de } \\
\text { elementos }\end{array}$ & Elementos & Elementos descriptores \\
\hline \multirow{7}{*}{$\begin{array}{l}\text { Elementos } \\
\text { duros }\end{array}$} & \multirow{3}{*}{ Estrategia } & Comunicación externa: medios \\
\hline & & Misión \\
\hline & & Visión \\
\hline & Estructura & Tipo de estructura organizacional \\
\hline & \multirow{3}{*}{ Sistema } & Innovación \\
\hline & & Logística \\
\hline & & Calidad \\
\hline \multirow{7}{*}{$\begin{array}{l}\text { Elementos } \\
\text { suaves }\end{array}$} & Estilo de administración & Estilo de liderazgo \\
\hline & \multirow{3}{*}{ Personal } & Comunicación interna: formal e informal \\
\hline & & Capacitación del personal: ámbito \\
\hline & & Medición de clima organizacional \\
\hline & Habilidades & Motivación \\
\hline & \multirow[t]{2}{*}{ Valores compartidos } & $\begin{array}{l}\text { Establecimiento de elementos de la cultura } \\
\text { organizacional }\end{array}$ \\
\hline & & Trabajo en equipo \\
\hline
\end{tabular}

Fuente: Investigación propia 
Loja (Ecuador), capital de la provincia del mismo nombre, cuenta según datos de la Superintendencia de Compañías (2015) con un aproximado de 681 empresas activas, de las cuales el 7\% corresponden al sector industrial, destacándose la producción de alimentos y bebidas como productos principales.

Al clasificarlas por su tamaño, el sector industrial tiene una participación del 76\% entre micro y pequeñas empresas (entre 3 y 24 trabajadores por empresa) y cuyos productos son consumidos a escala local y regional. Las medianas empresas corresponden el 21\% (entre 2 y 86 trabajadores por empresa), sus productos son consumidos a nivel local, regional y nacional. Loja cuenta además, con una única empresa catalogada como grande (aproximadamente 236 trabajadores) cubriendo los mercados nacional e internacional para la venta de sus productos

Las empresas estudiadas de acuerdo a las actividades que realizan según la Clasificación Internacional Industrial Unificada - CIIU y a su constitución se clasifican según lo indica el Gráfico 1.

\section{Gráfico 1. Constitución y actividad}

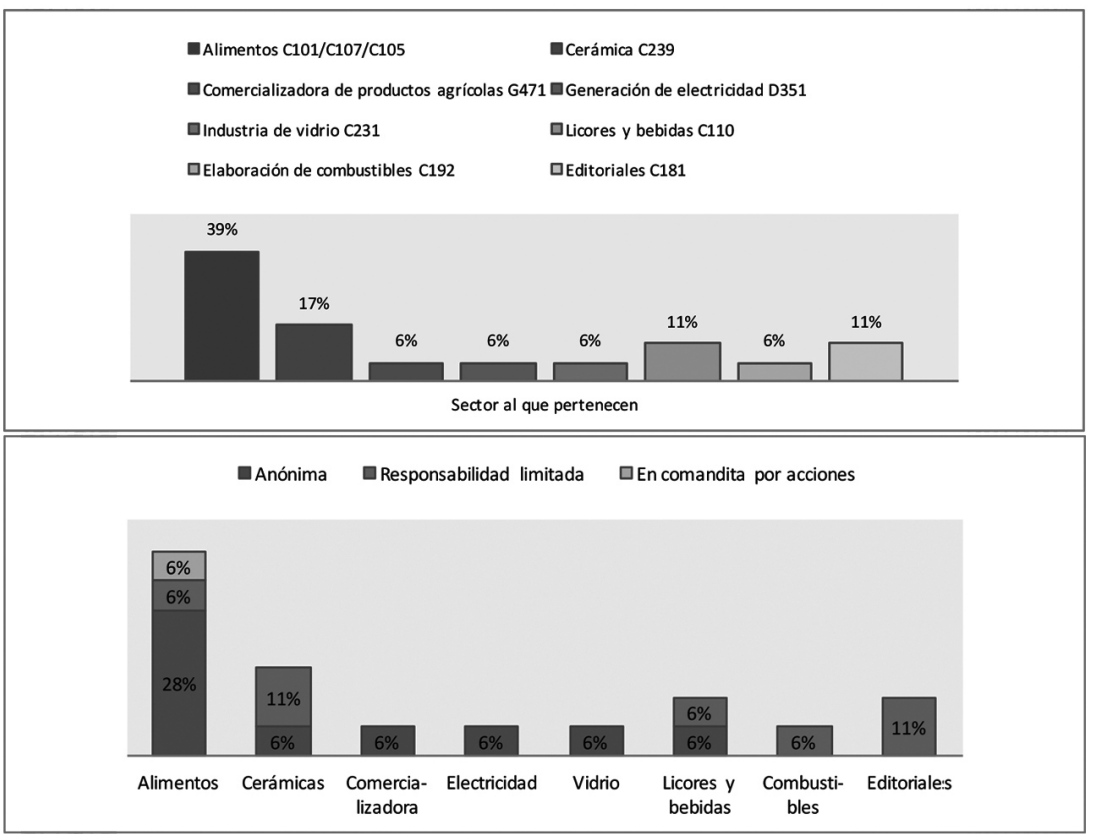

Fuente: Elaboración propia 
Las características de los elementos del modelo de las $7 \mathrm{~S}$ de Mckinsey, encontradas en las empresas industriales se presentan a continuación:

Resultados de la variable "Estrategia"

Gráfico 2. Características del elemento estrategia

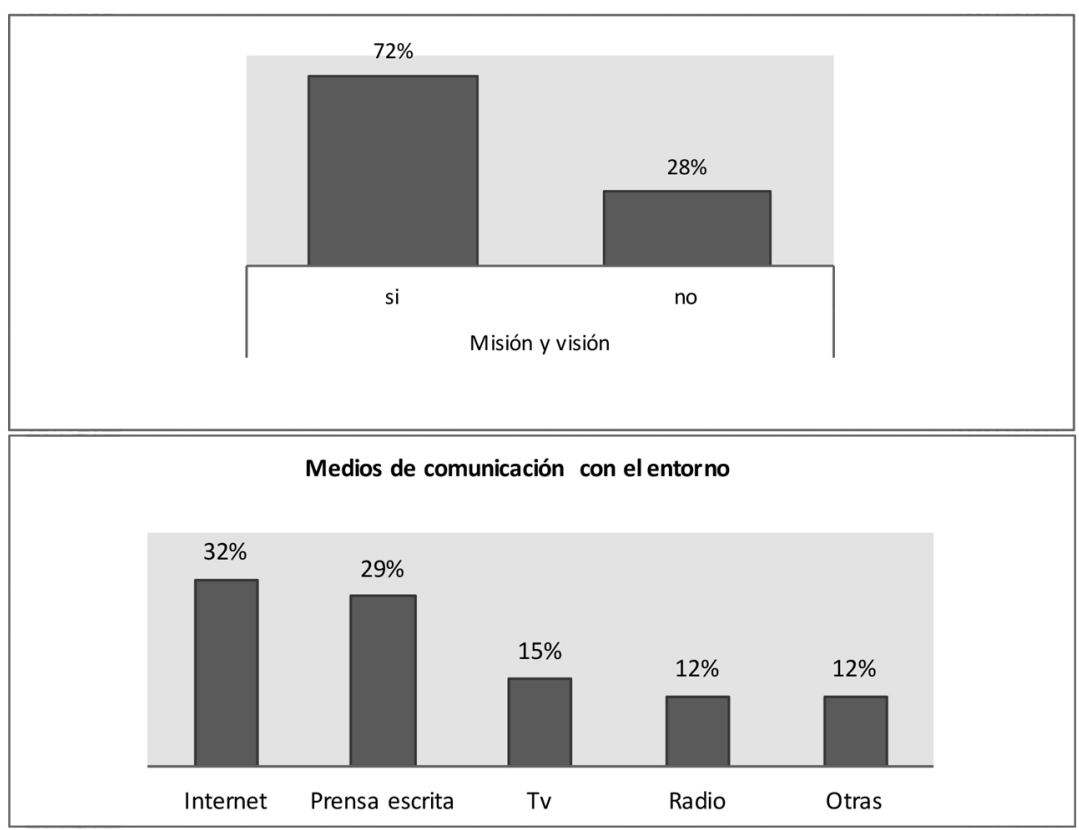

Fuente: Elaboración propia

En lo correspondiente a este elemento, se las puede resumir en que el $72 \%$ de las empresas poseen misión y visión definida, mientras que el restante $28 \%$ de las empresas no lo han definido. En lo correspondiente a comunicación con el entorno, todas las empresas indican realizarla a través de diferentes medios, siendo los más utilizados Internet y la prensa escrita.

Resultados de la variable "Estructura" 


\section{Gráfico 3. Características del elemento estructura}

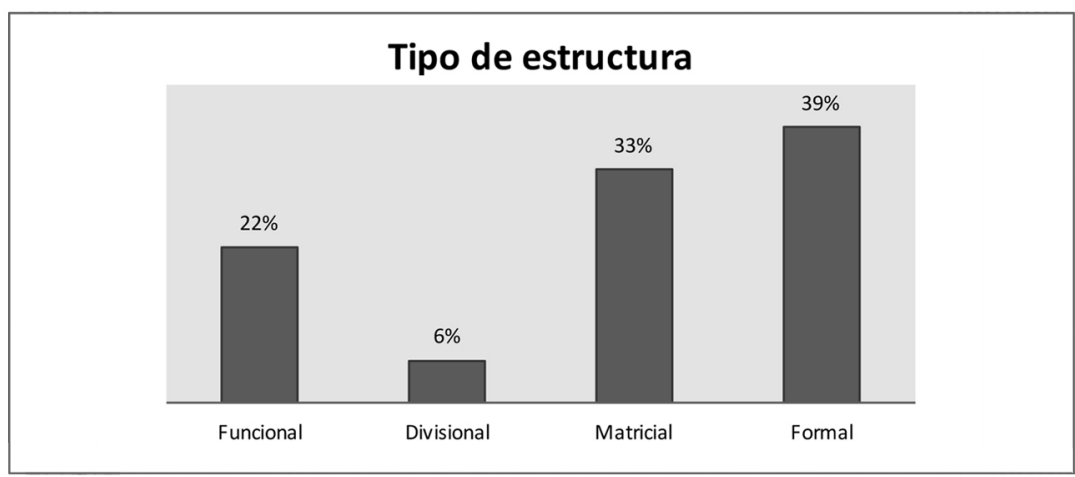

Fuente: Elaboración propia

En lo referente al tipo de estructura organizacional, todas las organizaciones reconocen una estructura formal definida, sin embargo solamente el $61 \%$ de las empresas han logrado identificar el tipo de estructura existente, clasificándose el 33\% como matricial, el 22\% como funcional y el $6 \%$ divisional.

\section{Resultados de la variable "Sistema"}

\section{Gráfico 4. Características del elemento sistema}

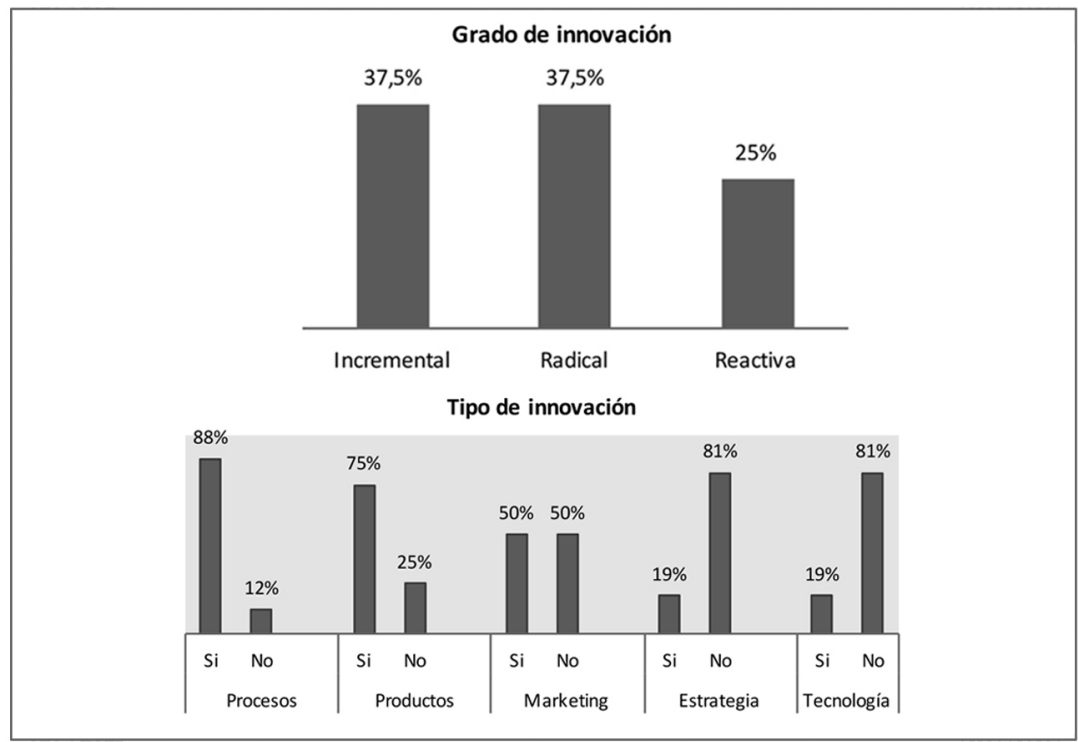




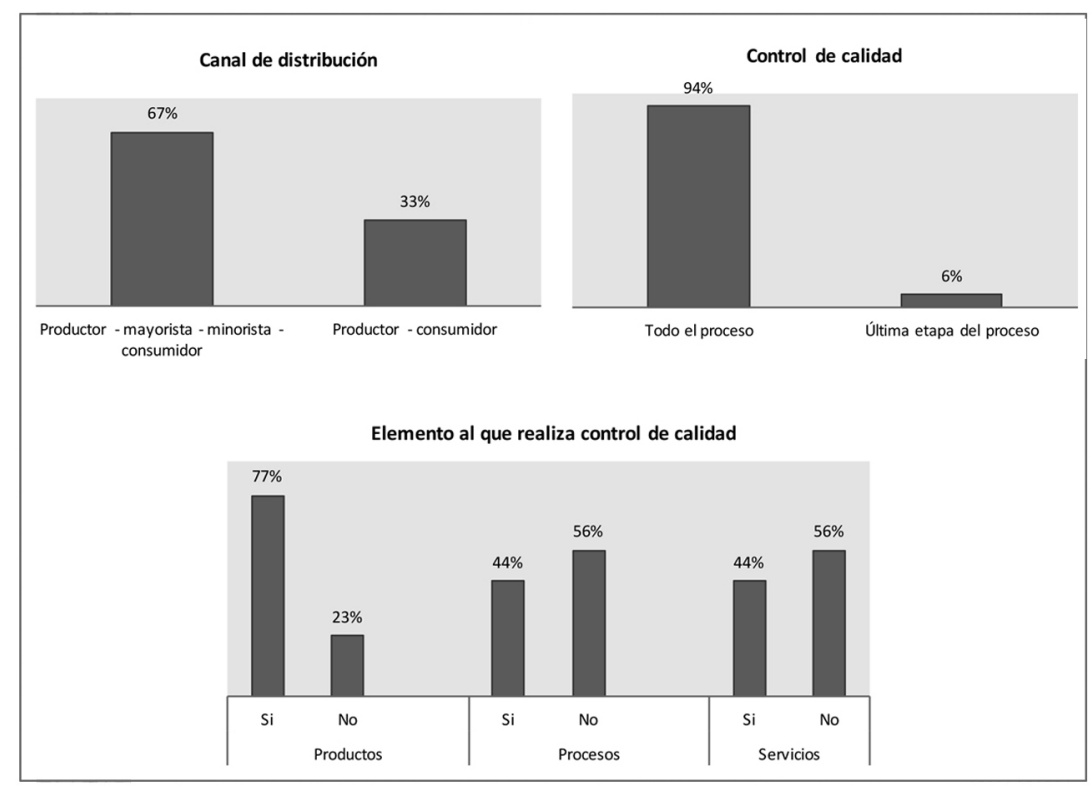

Fuente: Elaboración propia

Las características investigadas dentro de este elemento, corresponden a:

- Innovación: El 89\% de las empresas realizan procesos de innovación. Para estas empresas el grado de innovación más común es el incremental y el radical con $37,8 \%$ cada uno y la innovación reactiva el $25 \%$. El $88 \%$ de las empresas realizan innovación aplicada a procesos, el $75 \%$ a productos, el $50 \%$ a elementos de marketing. Mientras que se aplica innovación a estrategia y a tecnología en solo 19\% de la muestra.

- Con respecto a la distribución de productos, el $67 \%$ de las empresas utiliza el canal productor - mayorista - minorista y consumidor; mientras que el canal directo (productor - consumidor) solamente es aplicado por el $33 \%$.

- Calidad: Todas las organizaciones realizan control de calidad. El 94\% lo realizan a lo largo de todo el proceso y el $6 \%$ en la última etapa del proceso de producción. También se consultó respecto del elemento al que se realiza el control de calidad, emergiendo que el $77 \%$ lo realiza al producto terminado.

Resultados de la variable "Estilo de administración" 
Gráfico 5. Características del elemento estilo de administración

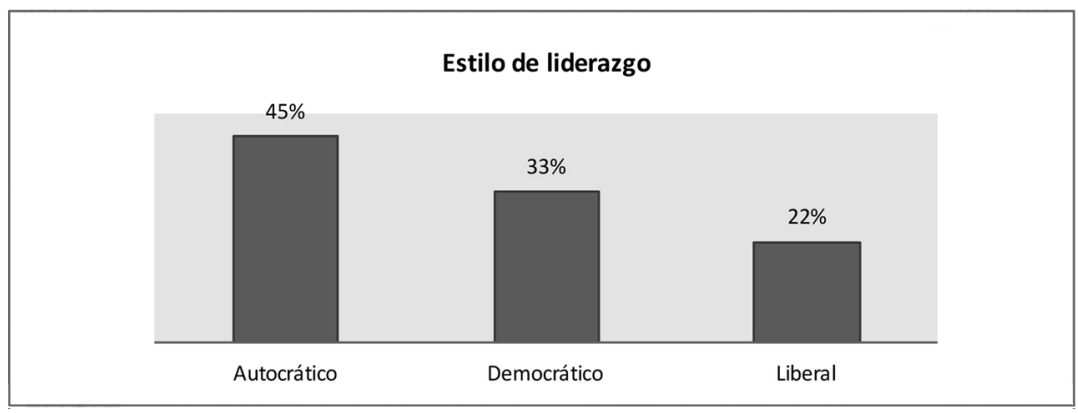

Fuente: Elaboración propia

Los tres estilos de liderazgo son practicados por las empresas del sector en estudio. El 45\% mantienen un estilo autocrático, caracterizado por imposición de órdenes y la capacidad de otorgar o denegar premios o castigos; el 33\% es de estilo democrático, que se caracteriza por consultar a subordinados y el $22 \%$ liberal que se refiere a quienes hacen uso reducido de su poder, otorgando a sus subordinados alto grado de independencia, de acuerdo a las características señaladas por Kootz \& Weihrich (2004).

\section{Resultados de la variable "Personal"}

\section{Gráfico 6. Características del elemento personal}

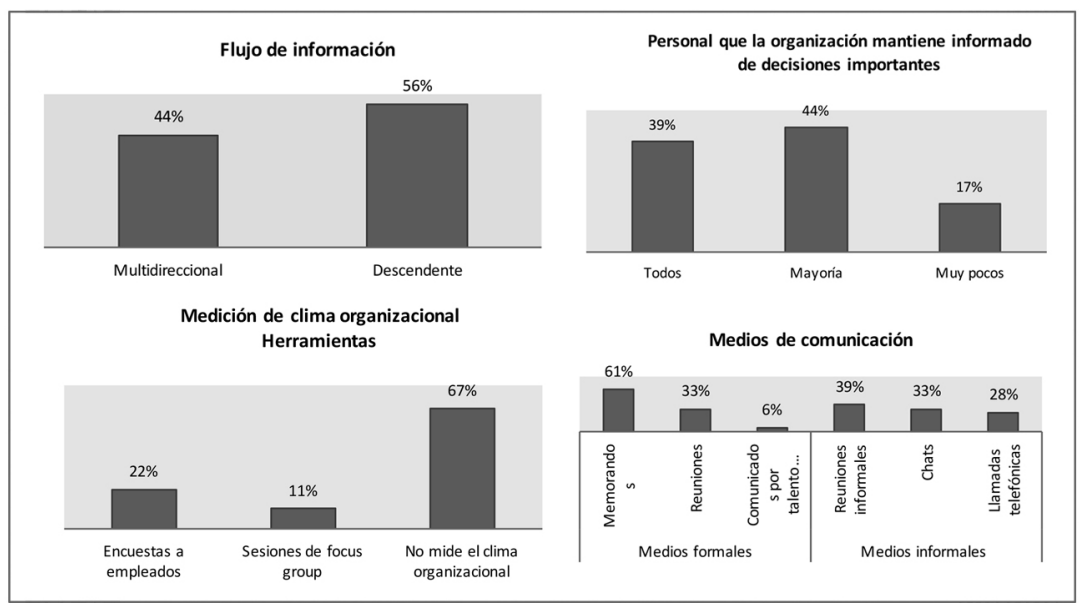




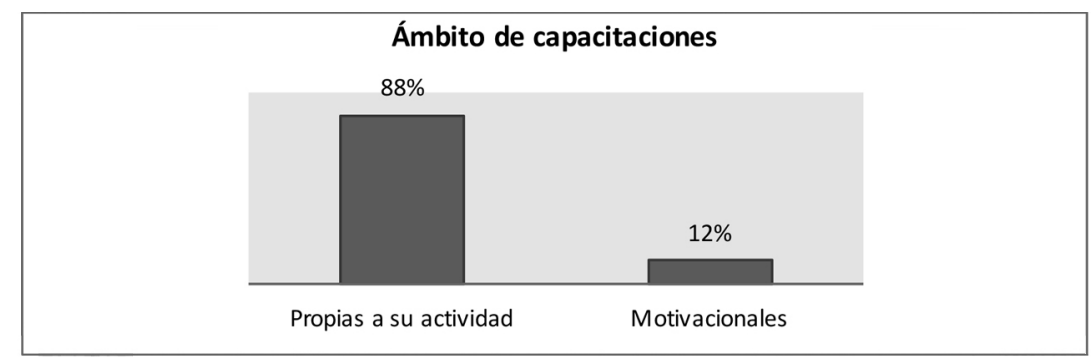

Fuente: Elaboración propia

- Flujo de información: el 56\% se caracteriza por realizarla de manera descendente y $44 \%$ de manera multidireccional. Sobre cuestiones trascendentales, las empresas en un $44 \%$ informan a la mayoría de sus colaboradores, el $39 \%$ a todos y el $17 \%$ a muy pocos.

- Las empresas no realizan medición del clima organizacional en el $67 \%$ de los casos. El 22\% se apoyan en encuestas para medirlo y el 11\% lo realizan a través de focus group.

- Los medios de comunicación interna formales más utilizados son los memorandos $(61 \%)$ y las reuniones formales $(33 \%)$. Por otro lado, los medios de comunicación informales utilizados son las reuniones informales (39\%), chats (33\%) y llamadas telefónicas internas (28\%).

- Capacitaciones: Todas las empresas de la muestra efectiva capacitan a sus empleados. El 88\% lo realiza en aspectos propios de su actividad y el 12\% en temas motivacionales, que se refieren principalmente a aspectos que fortalezcan el aspecto humano y de logro de objetivos de los colaboradores.

\section{Resultados de la variable "Habilidades"}

\section{Gráfico 7. Características del elemento habilidades}

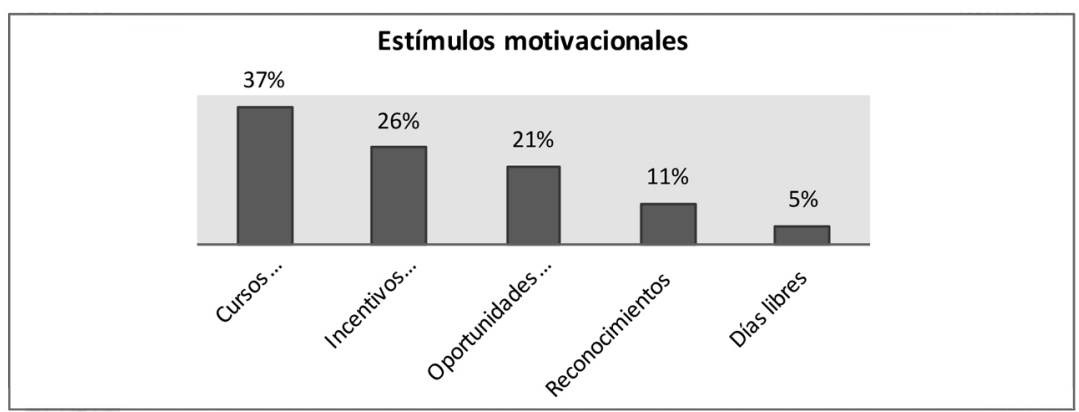

Fuente: Elaboración propia 
Caracterizado por los estímulos motivacionales como práctica de las organizaciones, las empresas recurren a los cursos y capacitaciones $37 \%$ como parte de motivación a sus empleados, el $26 \%$ a incentivos económicos, el $21 \%$ a aspectos relacionados a oportunidad de desarrollo, el $11 \%$ a reconocimientos y el $5 \%$ otorgan días libres.

Elemento: Valores compartidos

Gráfico 8. Características del elemento valores compartidos

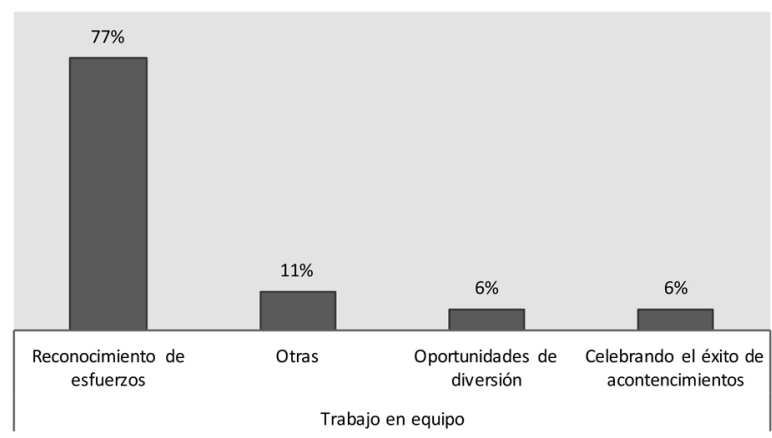

Elementos de la cultura organizacional

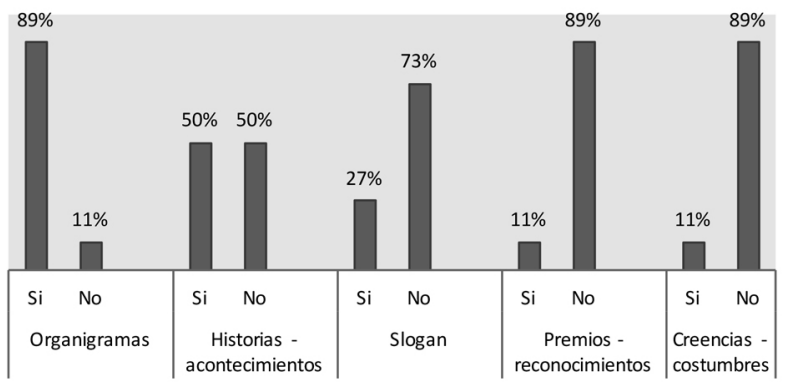

Fuente: Investigación propia

En lo referente a este elemento, se ha consultado respecto a los diferentes elementos de la cultura organizacional. Es así que el $89 \%$ de las empresas poseen organigramas como forma de estructurar y formalizar las tareas que se desarrollan, el $50 \%$ se apoya en historias y acontecimientos de las empresas, el $27 \%$ en el eslogan que los identifica y el $11 \%$ en aspectos referentes a premios y reconocimientos y creencias y costumbres. 
El trabajo en equipo fue el valor que todas las empresas practican, de los cuales el $77 \%$ lo realizan como una forma de reconocimiento de esfuerzos y el $6 \%$ como una forma de oportunidad de diversión y de celebrar el éxito.

\section{Discusión y conclusiones}

En la siguiente tabla se muestra la combinación de capacidades y características de las empresas sujetas al estudio que generaron variabilidad positiva en utilidad y en impuesto a durante el periodo 2013- 2014, así como la combinación de capacidades de aquellas que presentan variabilidad negativa.

Tabla 4. Características de los elementos del modelo de Mckinsey de las empresas de acuerdo a su rendimiento

\begin{tabular}{|c|c|c|c|c|c|c|}
\hline \multicolumn{2}{|c|}{ 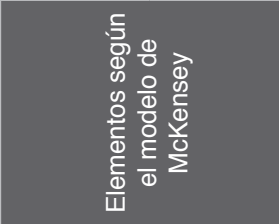 } & 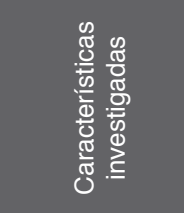 & 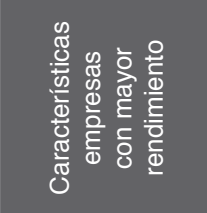 & 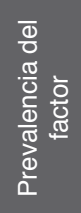 & 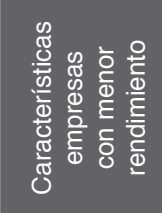 & 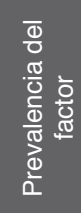 \\
\hline \multirow{10}{*}{$\begin{array}{l}\text { Elementos } \\
\text { duros }\end{array}$} & \multirow{3}{*}{ Estrategia } & $\begin{array}{l}\text { Comunica- } \\
\text { ción externa: } \\
\text { medios }\end{array}$ & Internet & $100 \%$ & Internet & $75 \%$ \\
\hline & & Misión & Definida & $100 \%$ & Definida & $100 \%$ \\
\hline & & Visión & Definida & $100 \%$ & Definida & $100 \%$ \\
\hline & Estructura & $\begin{array}{l}\text { Tipo de estruc- } \\
\text { tura organiza- } \\
\text { cional }\end{array}$ & Matricial & $50 \%$ & Formal & $75 \%$ \\
\hline & \multirow{6}{*}{ Sistema } & \multirow[b]{3}{*}{ Innovación } & Productos & $100 \%$ & Productos & $100 \%$ \\
\hline & & & Procesos & $50 \%$ & Procesos & $75 \%$ \\
\hline & & & $\begin{array}{l}\text { Tipo de innova- } \\
\text { ción: incremental }\end{array}$ & $50 \%$ & $\begin{array}{l}\text { Tipo de } \\
\text { innovación: } \\
\text { Reactiva }\end{array}$ & $50 \%$ \\
\hline & & Logística & $\begin{array}{l}\text { Productor - ma- } \\
\text { yorista - minoris- } \\
\text { ta - consumidor }\end{array}$ & $100 \%$ & $\begin{array}{l}\text { Productor } \\
\text { - mayorista } \\
\text { - minorista - } \\
\text { consumidor }\end{array}$ & $100 \%$ \\
\hline & & \multirow{2}{*}{ Calidad } & Productos & $100 \%$ & Productos & $75 \%$ \\
\hline & & & Procesos & $50 \%$ & Procesos & $75 \%$ \\
\hline
\end{tabular}




\begin{tabular}{|c|c|c|c|c|c|c|}
\hline \multicolumn{2}{|c|}{ 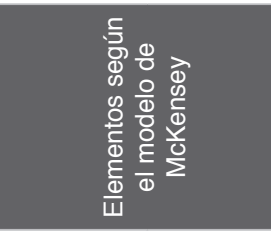 } & 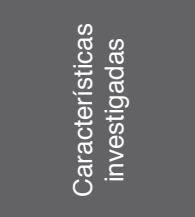 & 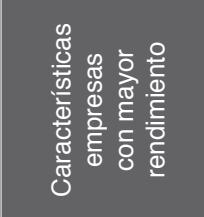 & 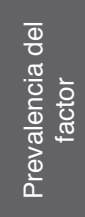 & 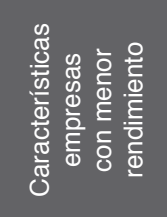 & 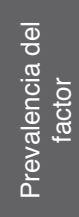 \\
\hline \multirow{9}{*}{$\begin{array}{l}\text { Elementos } \\
\text { suaves }\end{array}$} & \multirow{2}{*}{$\begin{array}{l}\text { Estilo de } \\
\text { adminis- } \\
\text { tración }\end{array}$} & \multirow{2}{*}{$\begin{array}{l}\text { Estilo de lide- } \\
\text { razgo }\end{array}$} & \multirow[b]{2}{*}{ Autocrático } & \multirow[b]{2}{*}{$75 \%$} & Democrático & $50 \%$ \\
\hline & & & & & Liberal & $50 \%$ \\
\hline & \multirow{4}{*}{ Personal } & \multirow{2}{*}{$\begin{array}{l}\text { Comunicación } \\
\text { interna: formal } \\
\text { e informal }\end{array}$} & $\begin{array}{l}\text { Memorandos } \\
\text { - reuniones } \\
\text { informales } \\
\end{array}$ & $75 \%$ & $\begin{array}{l}\text { Memorandos } \\
\text { - reuniones } \\
\text { informales } \\
\end{array}$ & $50 \%$ \\
\hline & & & $\begin{array}{l}\text { Flujo de informa- } \\
\text { ción: descen- } \\
\text { dente }\end{array}$ & $100 \%$ & $\begin{array}{l}\text { Flujo de } \\
\text { información: } \\
\text { multidirec- } \\
\text { cional }\end{array}$ & $100 \%$ \\
\hline & & $\begin{array}{l}\text { Capacitación } \\
\text { del personal: } \\
\text { ámbito }\end{array}$ & $\begin{array}{l}\text { Propios de su } \\
\text { actividad }\end{array}$ & $75 \%$ & $\begin{array}{l}\text { Propios de } \\
\text { su actividad }\end{array}$ & $75 \%$ \\
\hline & & $\begin{array}{l}\text { Medición de } \\
\text { clima organiza- } \\
\text { cional }\end{array}$ & No mide & $75 \%$ & $\begin{array}{l}\text { Encuestas a } \\
\text { empleados }\end{array}$ & $50 \%$ \\
\hline & $\begin{array}{l}\text { Habilida- } \\
\text { des }\end{array}$ & Motivación & $\begin{array}{l}\text { Incentivos eco- } \\
\text { nómicos, días } \\
\text { libres }\end{array}$ & $\begin{array}{l}\text { no } \\
\text { existe }\end{array}$ & $\begin{array}{l}\text { Incentivos } \\
\text { económicos }\end{array}$ & $50 \%$ \\
\hline & Valores & $\begin{array}{l}\text { Establecimiento } \\
\text { de elementos } \\
\text { de la cultura } \\
\text { organizacional }\end{array}$ & $\begin{array}{l}\text { Oroganigramas } \\
\text { definidos }\end{array}$ & $75 \%$ & $\begin{array}{l}\text { Organigrama, } \\
\text { misión visión, } \\
\text { historias y } \\
\text { aconteci- } \\
\text { mientos }\end{array}$ & $100 \%$ \\
\hline & & $\begin{array}{l}\text { Trabajo en } \\
\text { equipo }\end{array}$ & $\begin{array}{l}\text { Reconocimiento } \\
\text { de esfuerzos }\end{array}$ & $75 \%$ & $\begin{array}{l}\text { Reconoci- } \\
\text { miento de } \\
\text { esfuerzos }\end{array}$ & $50 \%$ \\
\hline
\end{tabular}

Fuente: Elaboración propia

Diversos estudios han señalado que existe una relación positiva entre "La implementación de estrategias prospectoras y analizadoras, el desarrollo de recursos y capacidades orientados a mercado y el vínculo de estos últimos con el crecimiento organizacional" (Ynzunza \& Izar, p. 2013), lo cual se alinea al hallazgo del presente estudio en lo referente a la innovación incremental en empresas de mayor rendimiento, en contraste con la innovación reactiva que existe en las empresas de menor rendimiento.

Por otro lado, se revela que "El nivel de productividad del trabajo es explicado por la calificación de los trabajadores, la dinámica del 
empleo está positivamente asociada a la existencia y formalidad del equipo de I+D" (Barletta, Pereira, Robert, \& Yoguel, 2013). Estos resultados se relacionan con el éxito de la alta capacitación del personal y la existencia de innovación tanto en procesos y productos.

En lo referente al estilo de administración se indica que "El liderazgo y sus buenas prácticas asociadas son un pilar fundamental en la construcción de la capacidad de innovación y esto puede cambiar dependiendo del sector en que se realice la investigación" (Bravo \& Herrera, 2009). El estilo de liderazgo autocrático para el caso del sector industrial investigado resulta ser parte de las buenas prácticas que sustentan el desarrollo de capacidades, sin embargo, el estilo que genera estas buenas prácticas podrá variar en el caso de otros sectores.

La organización, al ser un sistema abierto, está influenciada en forma permanente por una serie de factores de contingencia que se interrelacionan entre sí, y a la visión de sus directivos o administradores acerca de la forma cómo debe ser dirigida y organizada. Dichos aspectos como estrategia, estructura, sistema, estilo de administración, personal, habilidades y valores compartidos deben ser considerados al momento de aplicar estrategias que permitan el logro de objetivos y el rendimiento.

El modelo de las 7S de Mckinsey integra elementos que la organización necesita gestionar a fin de configurar la estrategia corporativa y ser exitosa, los cuales son: estrategia, sistemas y procedimientos, estructura, estrategia, estilo de gestión, habilidades y valores compartidos.

Las empresas, en su mayoría, poseen misión y visión como parte de su filosofía empresarial, así como mantienen una estructura formal que se refleja en la estructuración del trabajo a través de organigramas, además de realizar control de calidad en todo el proceso productivo.

El estilo del liderazgo que se aplica generalmente es de tipo autocrático y como consecuencia el flujo de información es descendente. Los diversos elementos estudiados revelan actuaciones distintas a las esperadas que difiere por la influencia sistémica del entorno. Así, las empresas estudiadas que presentan mayor rendimiento económico, muestran los siguientes elementos característicos:

- Generan estrategias y planes anticipados, mantienen una estructura organizacional matricial es decir un sistema de mandos múltiples que exige flexibilidad, en relación a los sistemas y procedimientos las organizaciones innovan en productos. 
- La logística se da a través del canal: producto, mayorista, minorista, consumidor, gestionan permanentemente la calidad en productos.

- El estilo de liderazgo que direcciona las organizaciones es autocrático por lo que el flujo de comunicación interna es descendente.

- El personal es capacitado en ámbitos propios a la actividad que desarrollan, no miden el clima laboral.

Las investigaciones futuras pueden enfocarse en estudiar cuáles otras capacidades presentan las organizaciones que están generando valor, así como identificar si la generación de valor es una consecuencia directa de las capacidades que las organizaciones han desarrollado. Estudios en estos ámbitos contribuirían a enriquecer la teoría administrativa y brindaría a los administradores mayores elementos al momento de tomar decisiones.

\section{Referencias}

Barletta, F., Pereira, M., Robert, V., \& Yogel, G. (2013). Argentina: Dinámica reciente del sector de software y servicios informáticos. Revista Cepal (110), 137-155. (goo.gl/pwrzoK) (2017-02-17).

Bravo, E., \& Herrera, L. (2009). Generación de capacidades dinámicas mediante la innovación organizacional: Un múltiple estudio de casos exploratorios. En: Actas del XIII Congreso de Ingeniería de Organización, 2-4 de septiembre 2009. Barcelona-Terrassa. (goo.gl/ffKKTM) (2017-02-17)

Daft, R. (2012). Management. Mason: Cencage Learning.

Ivanko, S. (2013) Modern Theory of Organization. Ljubljana: University of Ljubljana.

Koontz, H., \& Weihrich, H. (2004). Administración: una perspectiva global. México: McGraw-Hill.

Martínez, P., Pérez, A., \& Rodríguez del Bosque, I. (2014). Exploring the role of CSR in the Organizational Identity of Hospitality Companies: A Case from the Spanish Tourism Industry. Journal of Business Ethics, 124(1), 47-66. doi: https:// doi.org/10.1007/s10551-013-1857-1

O'Reilly, C., \& Tushman, M. (2013). Organizational Ambidexterity: Past Present and Future. Academy of Management Perspectives, 27(4), 324-338. doi: https://doi. org/10.5465/amp.2013.0025

Parsons, T. (1937). La estructura de la acción social. Madrid: Guadarrama.

Peters, T., \& Waterman, R. (1980). Structure is not organization. Business Horizons, 23(3), 14-26. doi: https://doi.org/10.1016/0007-6813(80)90027-0

Reis, G., \& Azebedo, M. C. (2015). Relations between organizational culture and authenticity: the authentic living in the organizational enviroment. RAM Revista de Administracao Mackenzie, 16(6) 48-70. doi: https://doi. org/10.1590/1678-69712015/administracao.v16n6p48-70 
Robbins, S. P., \& Coulter, M. (2005). Administración. Ciudad de México: Pearson Education.

Suárez-Hernández, J., \& Mirón, S.I. (2013). Teoría de los recursos y las capacidades. Un enfoque actual en la estrategia empresarial. Anales de estudios económicos y empresariales, (15), 63-89.

Superintendencia de Compañías (2015). Directorio de compañías registradas en la Superintendencia, al 27 de enero del 2015. Superintendencia de Compañias del Ecuador, Loja. (goo.gl/wALZzd) (2017-02-17).

Ynzunza, C., \& Izar, J. (2013). Efecto de las estrategias competitivas y los recursos y capacidades orientados al mercado sobre el crecimiento de las organizaciones. Contaduría y Administración, 58(1), 169-197. 